\title{
Representaciones sociales del
} concepto de empleo de habitantes de diversos contextos culturales $\mathrm{y}$ diferentes grupos poblacionales

\author{
Social representations of the employment concept of persons \\ of diverse cultural contexts and different population groups
}

Teresa Margarita Torres-López ${ }^{1}$ Jazmín Aranzazú Munguía-Cortés² y Antonio de Jesús De la Cruz-Villarreal ${ }^{3}$

Fecha de recepción: 2 de septiembre de 2020

Fecha de aceptación: 23 de noviembre de 2020

\begin{abstract}
1- Nacionalidad: Mexicana. Grado: Doctorado en Antropología Social y Cultural.. Adscripción: Universidad de Guadalajara. (iD ORCID: https://orcid.org/0000-0003-4157-5073. Correo electrónico: tere.torres.cucs@gmail.com

2- Nacionalidad: Mexicana. Grado: Doctorado en Ciencias de la Salud. Adscripción: Universidad de Guadalajara. (D) ORCID: https://orcid.org/0000-0001-7425-0676. Correo electrónico: jazminmunguia@gmail.com

3- Nacionalidad: Mexicana. Grado: Licenciatura en Psicología. Adscripción: Universidad de Guadalajara. (D) ORCID: https://orcid.org/0000-0002-7465-7844. Correo electrónico: ajcv90@gmail.com
\end{abstract}




\section{Resumen}

Este estudio compara el contenido de las representaciones sociales del concepto de empleo de habitantes de diversos contextos culturales (México, Colombia, Ecuador y España) y diferentes grupos poblacionales (estudiantes de bachillerato, estudiantes universitarios y población adulta). Participaron 690 personas, seleccionadas por muestreo propositivo, a las cuales se les aplicó la técnica de listados libres. El análisis fue por el método de análisis de contenido temático. Los diferentes grupos de participantes muestran coincidencias al representar el empleo con diferentes dimensiones como son el aspecto económico, los requisitos para obtenerlo, así como los beneficios personales y sociales derivados; $y$, en menor medida, los aspectos sociales y negativos del mismo. Se observaron algunas diferencias por los distintos contextos culturales y grupos de edad.

Palabras clave: cultura, representaciones sociales, empleo, jóvenes, adultos

\section{Abstract}

This study compares the content of the social representations of the concept of employment of inhabitants of different cultural contexts (Mexico, Colombia, Ecuador and Spain) and different population groups (high school students, university students and adult population). 690 people participated, selected by purposeful sampling, to whom the free listing technique was applied. The analysis was by the Thematic Content Analysis Method. The different groups of participants show coincidences when representing employment with different dimensions such as the economic aspect, the requirements to obtain it, as well as the derived personal and social benefits; and to a lesser extent, the social and negative aspects of it. Some differences were observed for different cultural contexts and age groups.

Keywords: culture, social representations, employment, youth, adults 


\section{Introducción}

$\mathrm{E}$ n 2019, la población mundial de 15 años y más (personas en su etapa productiva) era aproximadamente de 5,7 mil millones de hombres y mujeres, de este total, 2,300 millones (39\%) no estaban comprendidos en la fuerza laboral; 3,300 millones (57\%) tenían empleo y se calcula que 188 millones no contaba con trabajo remunerado (Oficina Internacional del Trabajo, 2020). En 2018, la brecha entre los géneros con respecto de la tasa de empleo era de $26 \%$, esto es, los hombres tenían una probabilidad 0,5 veces mayor de contar con empleo que las mujeres. En ese mismo año, la tasa de participación laboral de los jóvenes más elevada se registró en los países de menor ingreso (57\%), el valor más bajo (36\%) se registró en los países de ingreso mediano bajo (Oficina Internacional del Trabajo, 2019). En el presente estudio se utilizan dos conceptos: trabajo y empleo. De acuerdo al Tesauro de la Oficina Internacional del Trabajo (compilación de más de 4,000 términos relacionados con el mundo del trabajo) el primero se define como "el conjunto de actividades humanas, remuneradas o no, que producen bienes o servicios en una economía, o que satisfacen las necesidades de una comunidad o proveen los medios de sustento necesarios para los individuos". Mientras que empleo es "trabajo efectuado a cambio de pago y se refiere también, al número de personas bajo un régimen de autoempleo o empleo remunerado" (Oficina Internacional del Trabajo, 2018).

\subsection{El empleo y los trabajadores}

El trabajo se puede concebir como una fuente de identidad, sentido de pertenencia y crecimiento personal, además de que proporciona recursos económicos para las personas que cuentan con uno. Por ello el desempleo es un problema poblacional debido a sus efectos a nivel personal, económico y social (Moyano et al., 2013). El trabajo productivo es una actividad que además de generar recursos económicos tiene implicaciones psicosociales en la identidad, el sentido de utilidad, facilita el bienestar general y en lo personal genera bienestar psicológico de quienes lo realizan (Amador et al., 2019).

De acuerdo con Da Rosa et al. (2011) el estudio del significado del trabajo puede ubicarse en cinco diferentes corrientes epistemológicas: cognitivista, existencialista, construc-cionista, socio histórica y de estudios culturales. El principal punto de convergencia entre dichas perspectivas se refiere al papel de la cultura en "la producción de los sentidos y significados" (Da Rosa et al., 2011, p. 184). Por ello, la cultura y la sociedad son elementos fundamentales para la comprensión de las significaciones. Por su parte, Vesga (2017) considera que en el campo de los estudios sobre el trabajo es necesario considerar tres niveles: los hechos, lo psicológico y las conceptualizaciones teóricas. En los elementos de tipo psicológico, se considera que los seres humanos interpretan la realidad "haciendo representaciones de la misma y construyen significados para dar sentido a las experiencias que viven" (p. 93). Con lo cual se percibe, interpreta, comprende y significa la realidad en su totalidad, además de incluir las ideas que se tiene del sí mismo, todo dentro de un marco contextual histórico, social y cultural (Orejuela y Ramírez, 2011). 
Así, considerar tanto la cultura como el nivel psicológico de los trabajadores lleva a discutir sobre la centralidad del trabajo y del empleo dentro del contexto actual. De acuerdo con Sisto y Fardellla (2013, p. 128) "en las sociedades modernas el trabajo se ha utilizado como el principal articulador de la organización social”. Así, las personas integran su actividad en el proceso de producción social y a cambio de ello reciben no solo una remuneración, sino también reconocimiento social, ocupando así un rol fundamental en la configuración de la identidad, tanto individual como colectiva. Mas en la actualidad, la importancia del mismo se cuestiona. Según la Oficina Internacional del Trabajo (2019), en 2018, la mayoría de las 3,300 millones de personas empleadas en el mundo tuvieron déficits de bienestar material, de seguridad económica y de igualdad de oportunidades, y no tuvieron un margen suficiente de desarrollo humano. Es decir, que contar con empleo no siempre garantiza condiciones de vida dignas. Muchos trabajadores se sienten forzados a tener que aceptar puestos de trabajo que no son de su interés, suelen ser informales y mal remunerados, incluso tienen poco o nulo acceso a la protección social y a los derechos laborales.

\subsection{Propuesta teórica de acercamiento a las representaciones sociales del desempleo}

Las representaciones sociales constituyen una perspectiva teórica y metodológica que propone una aproximación al conocimiento del empleo dentro de un marco cultural y psicológico. Jodelet (2011) indica que son "una forma específica de conocimiento, de saberes ordinarios, incluidas en la categoría del sentido común y tienen como particularidad la de ser socialmente construidas y compartidas en el seno de diferentes grupos" (p. 134). Dentro de sus funciones prácticas, se apoyan en la experiencia de las personas, sirven de lectura de la realidad y de guía de actuación en la vida práctica y cotidiana. Las representaciones sociales se difunden por medio de los discursos y se manifiestan en las prácticas discursivas de la cotidianidad. Son compartidas, pero no son fijas, ya que pueden ser resignificadas por el intercambio individual y social, ya que las personas participan en su construcción, aportando su experiencia y conocimiento de sentido común (Sibaja, 2013). Las representaciones sociales son propias de los tiempos actuales debido a la gran cantidad de información existente, accesible por diversos medios masivos, por su vigencia relativamente breve (como son las opiniones) y por lo difícil que es estructurar tal cantidad de datos en un esquema teórico permanente. Así, el "sentido común se impone como la explicación más comprensible y determinante de las relaciones de intercambio social" (Mora, 2002, p. 23).

Una de las funciones sociales de las representaciones sociales es hacer habitual los objetos, personas y eventos que se encuentran en la vida cotidiana; con ello se establecen formas de conocimiento diferenciadas y a la vez compartidas por un grupo social y cultural determinado. Entre otras de sus funciones están las de favorecer la comunicación, fomentar el pensamiento colectivo y la identidad social. Además, permiten explicar las decisiones y conductas que se manifiestan en las relaciones sociales (Materán, 2008).

Existen dos enfoques para su estudio, uno es el procesual cualitativo y el segundo el enfoque estructural (con énfasis en el núcleo central de las representaciones sociales y los elementos periféricos). El 
enfoque procesual identifica a las representaciones sociales como procesos discursivos, caracteriza "al ser humano como productor de sentidos y focaliza su análisis en las producciones de significados del lenguaje, a través de los cuales los seres humanos construyen su mundo" (Banchs, 2000, p. 3.6). En el enfoque estructural, los contenidos de las representaciones sociales son considerados como estructuras organizadas, se caracteriza por identificar su estructura o su núcleo y por desarrollar explicaciones acerca de las funciones de esta estructura (Abric, 2001). El primero de ellos utiliza un enfoque metodológio cualitativo y el segundo cuantitativo.

\subsection{Las representaciones sociales del trabajo y el empleo}

En fechas recientes son varios los estudios sobre la conceptualización del empleo que han tenido como base el enfoque de las representaciones sociales. Algunos se realizaron en países latinoamericanos (México, Argentina, Brasil y Venezuela) y otros en países europeos (España y Francia). Las poblaciones objetivo han sido de estudiantes de nivel medio a universitario, y también han incluido a población adulta. Los que se han centrado en población más joven son los de Cerrato et al. (2003), Pérez-Rubio (2004), Kornbilt (2004), Larrañaga et al. (2007) y Scheffer-Garay (2011).

Todos estos destacan al empleo como un recurso para obtener un salario, bienes materiales, crecimiento personal, acceder a un espacio social y afectivo (Kornbilt, 2004). Estas representaciones sociales pueden variar según el género de los participantes (Cerrato et al., 2003) o por el contexto social donde viven, como en el caso de adolescentes en condiciones de riesgo, quienes visualizan en forma negativa su posible inserción laboral (Scheffer-Garay, 2011).

Los estudios sobre las representaciones sociales de empleo con estudiantes universitarios y adultos jóvenes son los de Longo (2003), Márquez et al. (2005), Navarro (2007), Methivier (2012) y Pereira (2013). En todos se destaca la importancia del empleo en la generación de recursos económicos, el ser una fuente de identidad, de estatus social. Es un aval de seguridad y de derechos sociales (Márquez et al., 2005; Longo 2003; Pereira, 2013), un medio para el logro de múltiples fines, la satisfacción de necesidades, asumir responsabilidades de adultos (Pereira, 2013), lograr la independencia económica (Navarro, 2007) y la realización personal (Longo 2003). Aunque reconocen la existencia de temores al buscar un empleo (Methivier, 2012).

Solo dos estudios consideran a la población adulta (Cerrato et al., 2003 y Pérez-Rubio, 2004). Las representaciones sociales de este grupo si bien también destacan la importancia de los aspectos económicos, agregan la realización personal, la dignidad y la identidad que se obtienen con la realización de un empleo. En el caso de población argentina sus representaciones sociales priorizan la importancia de las personas en su vínculo con el trabajo, y en el desarrollo personal y la dignificación (PérezRubio, 2004). Mientras que los españoles con más de 50 años de edad (socializados en un tipo de sociedad industrial) comparten una visión del trabajo de tipo social e identitaria (Cerrato et al., 2003).

El objetivo del presente documento es comparar el contenido de las representaciones sociales del concepto de empleo de población de diversos contextos y diferentes grupos poblacionales con el enfoque procesual cualitativo de las representaciones sociales. 


\section{Método}

\subsection{Diseño del estudio}

Estudio descriptivo comparativo de seis estudios de representaciones sociales (Torres-López y DíazVillanueva 2016; Torres-López et al., 2017; Torres-López et al., 2018a; Torres-López et al., 2018b; TorresLópez et al., 2018c; Torres-López et al., 2018d) y uno de concepciones culturales (Torres-López et al., 2019) de los conceptos de empleo y desempleo de habitantes de diversos contextos (México, Colombia, Ecuador y España) y diferentes grupos poblacionales (estudiantes de bachillerato, estudiantes universitarios y población adulta). Se incluyó el análisis de la primera fase de cada uno de los estudios mencionados en la intención de comprender el contenido de las concepciones culturales y las represen-taciones sociales del concepto de empleo.

El diseño comprendió la aplicación de técnicas asociativas, las cuales son señaladas como una de las formas de acceder al contenido de las representaciones sociales (Ruiz et al., 2001). Dichas técnicas favorecen la expresión natural de las personas, con lo que se espera que las respuestas sean libres de "racionalizaciones, sesgos de defensa o deseabilidad social" (Ruiz et al., 2001, p. 118), así se tiene un tipo de técnica proyectiva que permite acceder en forma más fácil y rápida que una entrevista a "los elementos que constituyen el universo semántico del término u objeto estudiado" (Ruiz et al., 2001, p. 118) y puede ser utilizada desde una perspectiva cualitativa.

\subsection{Contextos de estudio}

Los contextos de estudio fueron Guadalajara y Morelia de México; Bogotá y Cali de Colombia; Quito de Ecuador; Santa Cruz de Tenerife y San Sebastián, País Vasco de España. Los datos se levantaron en forma secuencial a lo largo de los años 2015 al 2017 (iniciando en México, luego en España, Ecuador y al final en Colombia).

$\mathrm{Al}$ respecto de estos lugares, se tiene información de América Latina y el Caribe en el año 2016, la tasa de ocupación era de 56,7\%, y la de desocupación de 8,2\%, en el grupo de desocupados el 38,9\% tenían entre 15 y 24 años (OIT, 2017b, p. 41). Específicamente en los países Andinos (Colombia, Ecuador y Perú) la tasa de ocupación: era de 62,7\%, y la de desocupación de 6,9\% (OIT, 2017b, p. 35). Mientras que en México la tasa de ocupación era de 57,3\%, y la de desocupación de 4,0\% (OIT, 2017b, p.36). La tasa de desocupación de los jóvenes aumentó para el promedio regional de 18,9\% en 2016 a 19,5\% en 2017. Es decir, que en toda la década, uno de cada cinco jóvenes estaba buscando empleo sin encontrarlo. Esto implicó un incremento de aproximadamente 9,8 a 10,2 millones en el número de jóvenes desocupados entre 2016 y 2017 (OIT, 2017b, p. 15). Los mayores incrementos de los salarios reales se observaron en el Cono Sur, seguido por los Países Andinos (Colombia, Ecuador y Perú) quienes tuvieron un aumento cercano al promedio regional, mientras que en Centroamérica y México, los incrementos salariales estuvieron muy por debajo del promedio, con crecimientos reales modestos (OIT, 2017b, p. 16). 
En el caso de España, en el año 2015 se reportó un repunte en el mercado laboral, el empleo aumentó un 3,1\% desde el comienzo hasta el final del tercer trimestre de dicho año. El aumento del empleo total fomentó los contratos temporales y registraba un lento incremento del número de nuevos contratos indefinidos (Comisión Europea, 2016a, p. 40). La nueva contratación permanente era limitada y "la proporción de trabajadores temporales era una de las más elevadas de la Unión Europea, sin que hayan aumentado las oportunidades de los trabajadores temporales para progresar hacia un contrato permanente" (Comisión Europea, 2016b, p. 10). El desempleo se redujo mientras que el desempleo juvenil era muy elevado, sobre todo en el caso de jóvenes sin trabajo ni estudios ni formación (Comisión Europea, 2016a, p. 40). En España en el año 2016 la tasa de desempleo era de 22,1\% (OIT, 2017a, p. 6).

\subsection{Muestreo y participantes}

En cada uno de los estudios se incluyó una muestra de participantes seleccionados por muestreo propositivo (Suri, 2011). El tamaño de la muestra fue con base en los estudios culturales, en los cuales dos elementos considerados son la competencia cultural esperada $(0,5)$ y el nivel de confianza aceptable $(0,95)$. Con base en esto se calcula un tamaño mínimo de 17 informantes (Weller, 2001) por cada contexto.

En el cuadro 1 se muestra el número de participantes en los estudios por contexto y grupo de edad. En el grupo de estudiantes de bachillerato ninguno reportó haber desarrollado actividades laborales remuneradas, en el grupo de estudiantes universitarios el 7\% dijo contar con empleo, y en el grupo de adultos la mayoría indicó tener trabajo, solo el 6\% estaba desempleado.

Cuadro 1

Participantes en los estudios por contexto y grupo de edad

\begin{tabular}{|c|c|c|c|c|}
\hline Participantes & México & España & Colombia & Ecuador \\
\hline $\begin{array}{c}\text { Estudiantes de } \\
\text { bachillerato de } 15 \text { a } \\
20 \text { años }\end{array}$ & $\begin{array}{c}76 \text { (36 mujeres y } 40 \\
\text { hombres) }\end{array}$ & $\begin{array}{c}120 \text { (62 mujeres y } \\
58 \text { hombres) }\end{array}$ & 80 (40 hombres y 40 \\
mujeres) & 80 (47 mujeres y 33 \\
hombres)
\end{tabular}

Fuente: elaboración propia a partir de los datos sociodemográficos obtenidos en los listados libres 


\subsection{Técnicas de obtención de información y análisis de los datos}

Se utilizó la técnica de listados libres a fin de obtener el contenido de las representaciones sociales del empleo. Se les pide a los participantes palabras o frases relacionadas con un tema específico: "Dime cinco palabras o frases que se te vienen la mente cuando escuchas: empleo"; después se les solicita que escriban una pequeña explicación de por qué dijeron cada una de ellas (Ruiz et al., 2001). Otros instrumentos utilizados fueron el cuestionario de pares (Abric, 2001) y pile sort (Bernard, 2006), los resultados de la aplicación de éstos dos últimos no se incluyen en el presente estudio.

Procedimientos de análisis: Partiendo de las definiciones que los participantes dieron a cada palabra o término en los listados libres, se realizó un análisis procesual, generando la construcción de categorías, a través de un análisis de contenido temático (Minayo, 2009) el cual busca identificar los núcleos de sentido dentro de las categorías emergentes, ello se realizó con el software especializado para el análisis de datos cualitativos Atlas.ti (Muhr, 1997).

\subsection{Calidad de la información}

En el enfoque procesual de las representaciones sociales se le otorga un lugar importante a la triangulación como una forma de garantizar la calidad de los datos, "combinando múltiples técnicas, teorías e investigadores para garantizar la confiabilidad en las interpretaciones" (Banchs, 2000, p. 3.8). La combinación de técnicas se llevó a cabo en los estudios previos antes citados (TorresLópez y Díaz-Villanueva 2016; Torres-López et al., 2017; Torres-López et al., 2018a; Torres-López et al., 2018b; Torres-López et al., 2018c; Torres-López et al., 2018d; Torres-López et al., 2019) donde se utilizaron las técnicas listados libres, cuestionario de comparación de pares y pile sort. En este caso al sólo presentarse el análisis procesual de la información obtenida con la primera técnica, se privilegió la triangulación por contrastación teórica y triangulación de investigadores (Flick, 2014). La contrastación teórica implica la comparación de los hallazgos con los obtenidos en los estudios citados en la introducción y la triangulación de investigadores se realizó en el proceso de análisis, donde participaron todos los investigadores en forma separada, llegando a los mismos resultados.

\subsection{Aspectos éticos}

Se garantizó a los participantes la confidencialidad y anonimato en la recolección y el análisis de la información. Antes de la aplicación de los instrumentos se explicaron los objetivos del estudio y se obtuvo el consentimiento informado verbal (Aguilera-Guzmán et al., 2008). El proyecto de investigación general que da origen a este estudio se titula "Concepciones culturales en torno a los conceptos de empleo y desempleo de diferentes grupos poblacionales", fue evaluado y aprobado por el Comité de ética, investigación y bioseguridad del Centro Universitario de Ciencias de la Salud, de la Universidad de Guadalajara, México (registro n CI02916). 


\section{Resultados}

\subsection{Representaciones sociales del empleo:}

En los cuadros 2, 3 y 4 se incluyen las definiciones de las representaciones sociales del empleo de cada grupo de participantes.

Cuadro 2

Representaciones sociales del empleo de estudiantes de bachillerato

\begin{tabular}{|c|c|}
\hline Guadalajara, México & San Sebastián, España \\
\hline Es una ocupación que requiere & Es de una ocupación que im- \\
contar con los elementos & plica acceso al dinero, favorece \\
necesarios tanto de prepa- & la satisfacción de necesidades, \\
ración académica, como de & genera felicidad, atención a la \\
valores (responsabilidad, & familia y posibilidad de futuro. \\
compromiso, honestidad, & Requiere de estudios (para \\
etc.) y esfuerzo personales & obtener un trabajo digno) y \\
(obligación, puntualidad, & responsabilidad, así como de \\
entre otros), lo que derivará & suerte, oportunidad, de ser un \\
en la obtención de un ingreso & milagro conseguirlo, el empleo \\
económico y de bienes mate- & es un privilegio y solo hay de \\
riales, además de beneficios & tipo temporal. Como elemen- \\
más en lo personal (emocio- & tos negativos resaltaron que \\
nes positivas, ser productivo, \\
exitoso e independiente, etc.) \\
hay poco, es difícil de conse- \\
yuir (lo que lleva a la angustia \\
en equipo, amigos, tolerancia, \\
etc.) Implica también algunos \\
aspectos negativos, como el \\
tiempo ocupado, la rutina, el \\
estrés y el cansancio. El énfasis \\
principal se encuentra en la \\
felicidad que genera y la res- \\
ponsabilidad requerida para su implica y esclavitud. Se le \\
realización.
\end{tabular}

Las representaciones sociales de los estudiantes de bachillerato de México y España coinciden en que el empleo es necesario para la obtención de un recurso económico que permite la satisfacción de necesidades, el crecimiento personal, genera emociones positivas y otros beneficios sociales; requiere de preparación y esfuerzos personales e incluyeron algunos aspectos negativos del mismo. La principal diferencia es que solo los estudiantes españoles señalan la escasez del empleo, la suerte u oportunidad para conseguirlo (sería un "milagro"), la asociación del término con el desempleo (conocido como "paro" en España) y los aspectos negativos fueron tanto individuales como sociales. Mientras que los mexicanos destacaron la felicidad que genera, así como la responsabilidad que implica y los aspectos negativos fueron de tipo individual. 
Cuadro 3

Representaciones sociales del empleo de jóvenes universitarios

\begin{tabular}{|c|c|c|}
\hline Guadalajara, México & Cali, Colombia & Quito, Ecuador \\
\hline $\begin{array}{l}\text { Es una ocupación que } \\
\text { requiere de aptitudes, } \\
\text { actitudes y energía per- } \\
\text { sonales para su realiza- } \\
\text { ción; comprende diversos } \\
\text { componentes de tipo eco- } \\
\text { nómico, genera beneficios } \\
\text { materiales producto de } \\
\text { la percepción económica, } \\
\text { (ganancias, comodidades } \\
\text { y prestaciones laborales), } \\
\text { más beneficios } \\
\text { personales (bienestar, } \\
\text { estabilidad, superación, } \\
\text { etc.) y sociales } \\
\text { (familia, socialización } \\
\text { y convivencia). Incluyó } \\
\text { como aspectos negativos: } \\
\text { genera cansancio, estrés } \\
\text { e implica mucho tiempo; } \\
\text { es escaso, mal pagado, } \\
\text { hay explotación de los } \\
\text { trabajadores, desigualdad } \\
\text { entre hombres y mujeres, } \\
\text { nepotismo e implica pagar } \\
\text { impuestos. }\end{array}$ & $\begin{array}{l}\text { Es una actividad laboral } \\
\text { que permite contar con se- } \\
\text { guridad económica y acce- } \\
\text { so a beneficios materiales. } \\
\text { Requiere de competencias } \\
\text { y valores de tipo personal } \\
\text { así como de contar con } \\
\text { la oportunidad. Implica } \\
\text { esfuerzo a fin de tener } \\
\text { crecimiento, superación, } \\
\text { estabilidad, progreso y, } \\
\text { por tanto, un futuro. Puede } \\
\text { generar frustración por la } \\
\text { dificultad para alcanzar } \\
\text { logros en el ámbito laboral, } \\
\text { así como la percepción de } \\
\text { esclavitud por exceso de } \\
\text { trabajo. Es una actividad } \\
\text { que se desempeña en } \\
\text { empresas o instituciones, } \\
\text { en las cuales se tendrá } \\
\text { la convivencia con otros } \\
\text { compañeros de trabajo. }\end{array}$ & $\begin{array}{l}\text { Es una ocupación y un } \\
\text { derecho que se realiza } \\
\text { a cambio de un salario, } \\
\text { requiere de experiencia, } \\
\text { conocimientos, compromi- } \\
\text { so, esfuerzo, motivación, } \\
\text { responsabilidad, deseos } \\
\text { de superación, además } \\
\text { de contar con suerte } \\
\text { para encontrarlo. Genera } \\
\text { estabilidad, bienestar, } \\
\text { autoridad, desarrollo, } \\
\text { dignidad e independen- } \\
\text { cia personales así como } \\
\text { la posibilidad de futuro, } \\
\text { éxitos y logros. Implica la } \\
\text { ocupación del tiempo, pue- } \\
\text { de ser agotador y generar } \\
\text { insatisfacción cuando no } \\
\text { es acorde a las necesidades } \\
\text { y expectativas propias. Se } \\
\text { le asocia al desempleo. }\end{array}$ \\
\hline
\end{tabular}

Fuente: elaboración propia a partir del análisis de contenido realizadoa las respuestas obtenidas en los listados libres

Las representaciones sociales sobre el empleo de los jóvenes universitarios de los tres contextos incluidos coinciden en señalar como una ocupación o actividad laboral que genera recursos económicos, beneficios personales (bienestar, estabilidad, superación, crecimiento, progreso, desarrollo, independencia y futuro) y sociales (apoyo a la familia, socialización, convivencia). Este grupo de edad es el que incluye más requerimientos para obtener un empleo como son aptitudes, actitudes, competencias, conocimientos, responsabilidad y valores de tipo personal. Agregaron además, aspectos negativos tanto individuales como sociales.

En cuanto a las diferencias, solo los jóvenes de Colombia visualizaron el espacio laboral en empresas o instituciones y los de Ecuador lo asociaron al desempleo. Además, tanto los ecuatorianos como los colombianos indicaron que es necesaria la oportunidad y la suerte para encontrar un trabajo remunerado. 


\section{Cuadro 4}

\begin{tabular}{|c|c|c|c|}
\hline Morelia, México & Tenerife, España & San Sebastián, España & Bogotá, Colombia \\
\hline $\begin{array}{l}\text { Es la realización de un } \\
\text { trabajo u ocupación } \\
\text { con lo que se obtiene } \\
\text { dinero para lograr } \\
\text { seguridad, estabili- } \\
\text { dad y tranquilidad, } \\
\text { requiere de responsa- } \\
\text { bilidad y oportunidad. } \\
\text { Genera felicidad, salud } \\
\text { y progreso económico } \\
\text { personal y familiar. Se } \\
\text { agregan otros elemen- } \\
\text { tos como la satisfac- } \\
\text { ción personal y que } \\
\text { implica mucho tiempo } \\
\text { para su desarrollo (10 } \\
\text { a } 12 \text { horas diarias). }\end{array}$ & $\begin{array}{c}\text { Es un oficio que per- } \\
\text { mite el acceso a dinero } \\
\text { para la satisfacción de } \\
\text { necesidades perso- } \\
\text { nales y familiares, de } \\
\text { bienes materiales. Tie- } \\
\text { ne efectos personales } \\
\text { y emociones positivas } \\
\text { y bienestar. Requiere } \\
\text { de responsabilidad, } \\
\text { compromiso, vocación, } \\
\text { formación académica } \\
\text { adecuada y suerte. Es } \\
\text { una oportunidad de } \\
\text { desarrollo personal y } \\
\text { profesional, permite } \\
\text { el compañerismo y } \\
\text { la integración social. } \\
\text { Se le asocia al paro o } \\
\text { desempleo y que pocas } \\
\text { personas pueden acce- } \\
\text { der a un trabajo. }\end{array}$ & $\begin{array}{l}\text { Es un trabajo u ocu- } \\
\text { pación que implica } \\
\text { acceso a recursos } \\
\text { económicos, es un } \\
\text { medio para obtener } \\
\text { dignidad, realización } \\
\text { y futuro, requiere } \\
\text { de responsabilidad, } \\
\text { implica horas para su } \\
\text { realización y es difícil } \\
\text { de obtener, promueve } \\
\text { la migración. Genera } \\
\text { cansancio, rutina, y es- } \\
\text { clavitud. Se encuentra } \\
\text { asociado a la crisis, la } \\
\text { precariedad en su des- } \\
\text { empeño (mal pagado, } \\
\text { malo) y al desempleo } \\
\text { o paro (sobre todo } \\
\text { en el caso de los más } \\
\text { jóvenes). }\end{array}$ & $\begin{array}{l}\text { Es una ocupación para } \\
\text { la obtención de dinero, } \\
\text { permite el bienestar, } \\
\text { estabilidad y felicidad, } \\
\text { requiere de contar } \\
\text { con responsabilidad. } \\
\text { Elementos adicionales } \\
\text { a ello son la conviven- } \\
\text { cia con compañeros, } \\
\text { permite el crecimiento } \\
\text { personal y la obten- } \\
\text { ción de prestaciones } \\
\text { como son las vacacio- } \\
\text { nes. Aspectos negati- } \\
\text { vos: genera explota- } \\
\text { ción, es insuficiente, } \\
\text { mal remunerado, con } \\
\text { pocos incentivos. car- } \\
\text { ga laborales pesadas, } \\
\text { implica gran parte del } \\
\text { día, además de que } \\
\text { hay mucho empleo } \\
\text { informal. }\end{array}$ \\
\hline
\end{tabular}

La población adulta de los cuatro contextos incluidos coincidió en representar al empleo como un trabajo, ocupación, oficio que genera la obtención de dinero para la satisfacción de necesidades personales y familiares. Incluyen los requisitos para obtenerlo y desarrollarlo, así como las efectos positivos en su crecimiento personal y su salud emocional. Las diferencias se ubican en que la población de Tenerife, España y de Bogotá, Colombia señalan la importancia de la convivencia con los compañeros de trabajo; también, los participantes mexicanos y españoles indicaron que es difícil encontrar un empleo por lo que se requiere oportunidad y suerte. Los cuatro grupos incluyeron aspectos negativos del empleo, solo que la población de San Sebastián, España y de Bogotá, Colombia fueron más enfáticos en ello. Los colombianos destacaron las condiciones de precariedad personales en que desarrollan su actividad laboral, mientras que los españoles se enfocaron más en problemas sociales derivados de empleos precarios. 


\subsection{Categorías de las representaciones sociales del empleo}

Las categorías para el análisis de contenido de la información obtenida en los listados libres son las mismas que se utilizaron en los estudios previos antes citados (Torres-López y Díaz-Villanueva 2016; Torres-López et al., 2017; Torres-López et al., 2018a; Torres-López et al., 2018b; Torres-López et al., 2018c; Torres-López et al., 2018d; Torres-López et al., 2019):

Componentes de empleo: “Términos asociados al desarrollo de una actividad laboral” (Torres-López et al., 2018b, p. 6). Labor, trabajo, ocupación, actividad, manufactura, tiempo, horario, jefe, negocio, empresa, empleado y obligación, etc.

- Componente económico: "Obtención de un salario para cubrir todo tipo de necesidades económicas” (Torres-López et al., 2018b, p. 6). Salario, remuneración, economía, oportunidad de ganar dinero, etc.

- Beneficios personales: "Consecuencias para la persona que realiza el trabajo" (Torres-López et al., 2018b, p. 6). Bienestar, felicidad, futuro, seguridad, estabilidad, tranquilidad, experiencia, gratificación, independencia, madurez, mejoría, desarrollo, superación, etc.

- Beneficios materiales: "Adquisiciones de bienes específicos producto del salario laboral" (Torres-López et al., 2018b, p. 6). Casa, automóvil, comida, consumo, comodidad, estatus, ganancias, pago de gastos, gustos, lujos, contar con prestaciones como vacaciones, etc.

- Elementos necesarios: "Requisitos para la realización de un trabajo" (Torres-López et al., 2018b, p. 6). Formación académica, conocimientos, compromiso, responsabilidad, madurez, esfuerzo, energía, puntualidad, ganas, etc.

- Aspectos sociales: "Componentes comunitarios del empleo" (Torres-López et al., 2018b, p. 6). Compañerismo, socialización, trabajo en equipo, desarrollar la tolerancia y convivir con diferentes tipos de personas, etc.

- Aspectos negativos: "Derivados de la realización de un determinado empleo" (Torres-López et al., 2018b, p. 6). Las ideas incluidas en ésta última categoría se agrupan en aspectos individuales y sociales. Entre los primeros incluyeron el tiempo ocupado (implica gran parte del día), el cansancio y agotamiento que genera (cargas laborales pesadas), la rutina, las emociones negativas que genera (estrés, angustia, insatisfacción), sobre todo cuando el empleo no es acorde a necesidades y expectativas propias. Los de tipo social son que no hay suficientes empleos, la precariedad de los trabajos remunerados (mal pagados, con pocos incentivos, empleo informal), la desigualdad entre hombres y mujeres, el pago de impuestos, el nepotismo, la explotación y esclavitud (por exceso de trabajo) y finalmente la asociación con el desempleo. 
En la gráfica 1 se presentan los porcentajes de las categorías de las representaciones sociales del empleo de jóvenes estudiantes de bachillerato, donde se observan las diferencias entre las respuestas de los dos grupos de población. La principal coincidencia es en la categoría de los beneficios personales, el resto de categorías muestra diferencias, entre las más notables están los componentes de empleo, los beneficios materiales y los aspectos negativos.

\section{Gráfica 1}

Categorías de las representaciones sociales del empleo de estudiantes de bachillerato

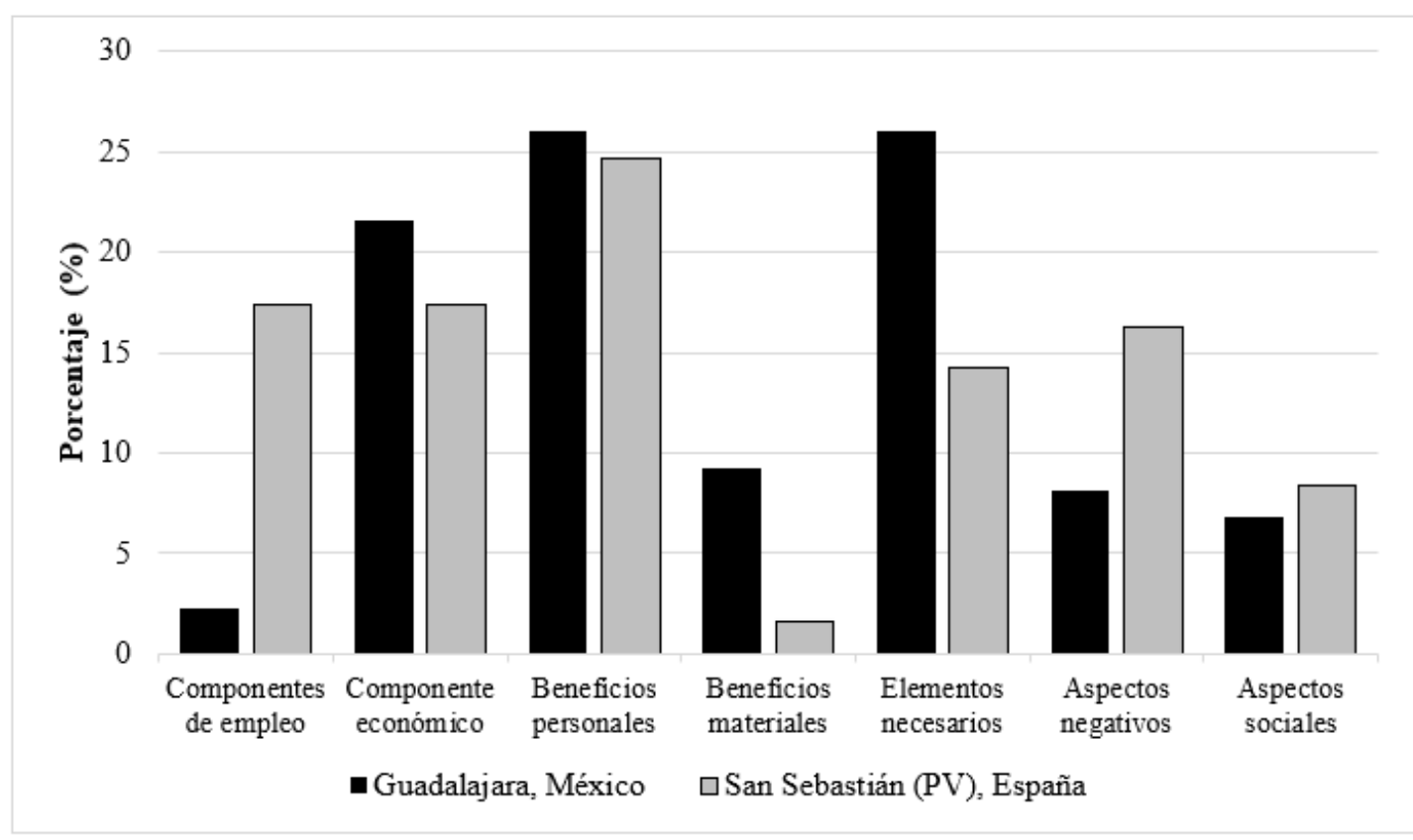

Fuente: elaboración propia a partir del análisis de contenido realizado a las respuestas obtenidas en los listados libres

En la gráfica 2 se presentan los porcentajes de las categorías de las representaciones sociales del empleo de jóvenes universitarios, donde puede observarse lo ya señalado sobre los requisitos para obtener un empleo, ya que es la categoría con más porcentaje de respuestas. La preparación académica fue mencionada en ello, esto ha sido resaltado como un aspecto central para el acceso al empleo y para el desempeño laboral. Llama la atención el que el componente económico no ocupa un lugar relevante al igual que los beneficios materiales (menor en el caso de los jóvenes ecuatorianos). Y los aspectos sociales solo fueron incluidos por los jóvenes mexicanos y colombianos. 


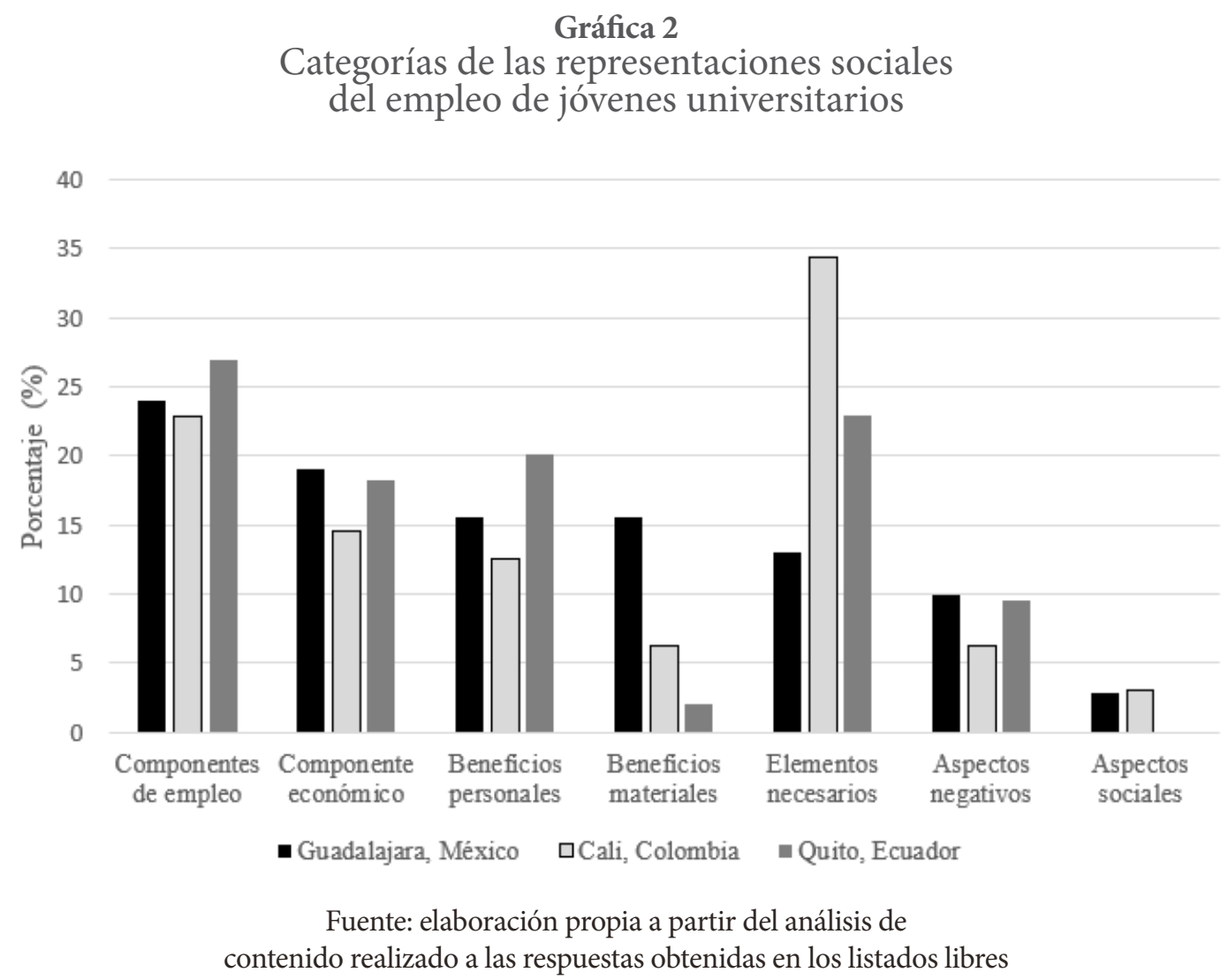

En la gráfica 3 se presentan los porcentajes de las categorías de las representaciones sociales del empleo de población adulta. Los cuatro grupos de población señalaron a los beneficios personales como los más relevantes, y en segundo lugar los componentes de empleo, excepto el grupo mexicano el cual ubicó en segundo lugar los elementos necesarios. El componente económico está ubicado en tercer lugar, mientras que los requerimientos para obtener un empleo son menores que el grupo de los jóvenes universitarios. 
Gráfica 3

Categorías de las representaciones sociales del empleo de la población adulta

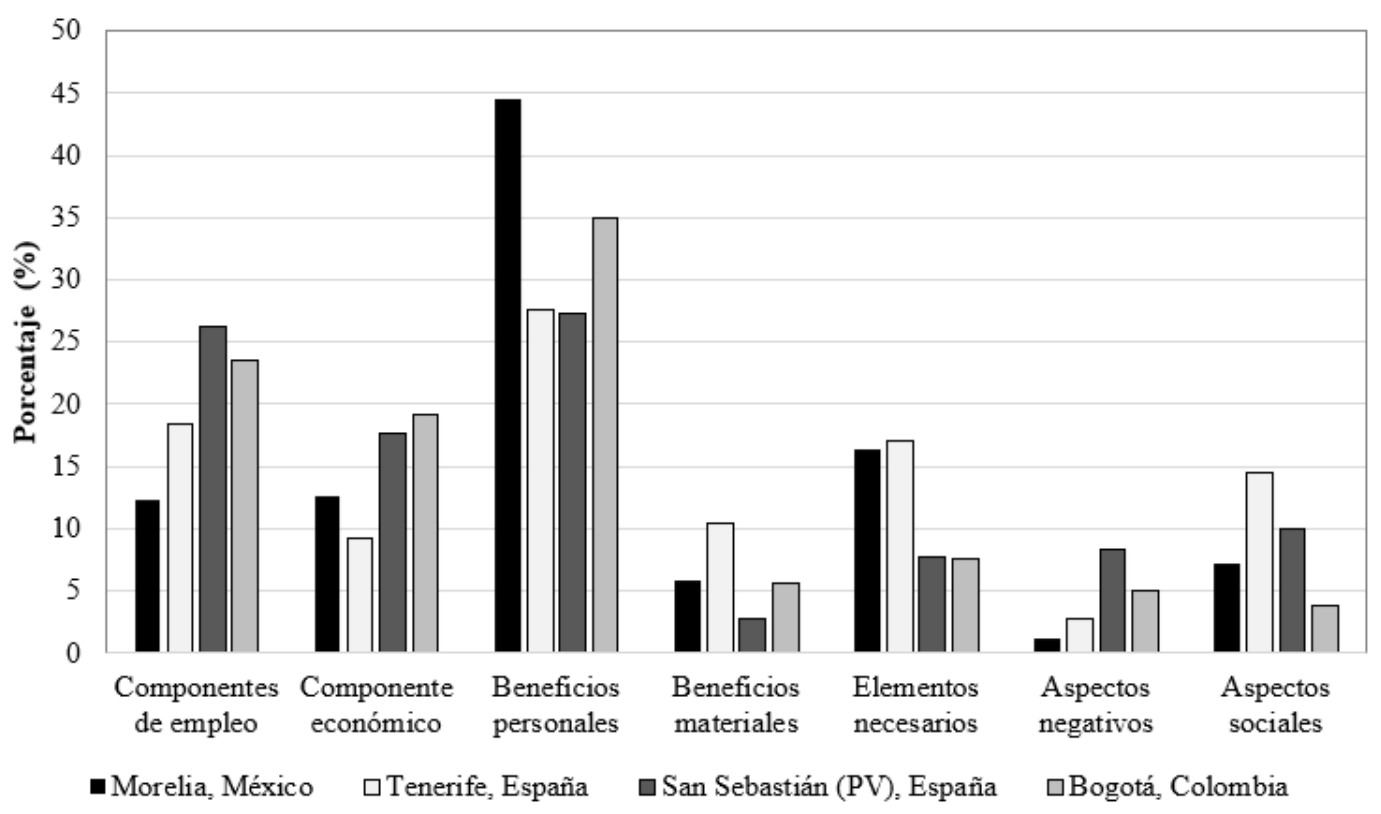

Fuente: elaboración propia a partir del análisis de contenido realizado a las respuestas obtenidas en los listados libres

\section{Discusión y conclusiones}

En todos los grupos de población participantes se encontró una visión global de las representaciones sociales del empleo que comprende diferentes dimensiones culturales y sociales de la misma. Los elementos ponen en relieve las ganancias económicas y personales, así como también aspectos negativos y sociales del empleo. Donde incluye al desempleo como un componente del empleo. Una de las funciones de las representaciones sociales es "hacer convencionales los objetos, personas y eventos que se encuentran en la vida cotidiana" (Materán, 2008, p. 245), en este caso las representaciones sociales del empleo.

Otra coincidencia es que ninguno de los participantes indicó el elemento económico en primer lugar, ya que fue incluido en segundo lugar en los grupos que ya han desarrollado actividades laborales remuneradas y en tercer lugar en las que no han tenido experiencia laboral. Lo que unido al señalamiento de los beneficios personales y emocionales de contar con un empleo, destaca que el empleo genera recursos económicos, ganancias psicológicas y sociales, además de fortalecer la identidad y la incorporación a la colectividad (Moyano et al., 2013; Serrano-Argüeso, 2015). Además, proporciona un sentimiento de pertenencia y de aportación social que beneficia a otras personas (Da Rosa et al., 2011).

En el grupo de estudiantes de bachillerato, aun cuando no han desarrollado actividades laborales remuneradas, presentaron una representación social del empleo con las mismas dimensiones encontradas en los otros grupos de participantes. Es probable que sus representaciones sociales partan de una lectura propia de su realidad y de las experiencias de otras personas o bien, que han desarrollado algún tipo de 
trabajo no remunerado o en condiciones precarias, así como de sus propias creencias sobre su realidad contextual. Con lo cual se logra la función comunicativa y creadora de conocimiento de las representaciones sociales, ya que una de sus bases es considerar a los seres humanos como generadores de conocimiento y no solo reproductores del mismo (Jodelet, 2011).

Los jóvenes universitarios resaltaron los requerimientos necesarios para la obtención de un empleo, entre otros la preparación académica, el tener conocimientos y experiencia, así como responsabilidad. Para ellos los estudios académicos forman parte del núcleo central de sus representaciones sociales, tanto para conseguir un empleo como para el desarrollo de su actividad laboral. En la actualidad a pesar del aumento en la cobertura escolar y el aumento de los años de escolaridad ya no es garantía de acceso a trabajos formales o de calidad, mas sí les favorece tener prioridad en relación con los jóvenes con menor escolaridad (Isacovich, 2015). Lo que se puede contextualizar con la información proporcionada por la Comisión Europea (2016a) y la OIT (2017a y 2017b) en el sentido que que los jóvenes son la poblaión más afectada por la falta de oportunidades para su inserción laboral en empleos de calidad, tanto en Latinoamérica como en España. Ejemplo de ello es un estudio realizado en México con estudiantes de una universidad pública, se encontró que los egresados tenían altas expectativas respecto de su inserción laboral, basadas en la autopercepción de contar con una buena preparación académica. Sin embargo, comprobaron en la realidad tener más dificultades de las esperadas para encontrar trabajo (Simón, 2015). Navarro (2007) indica el caso de jóvenes que cuentan con carrera universitaria ya no les garantiza un empleo de calidad, ya que en la selección de candidatos para empleos es usual que los empleadores consideren más la experiencia de trabajos anteriores que los estudios profesionales, o bien hay discrepancias entre la vocación, el oficio deseado y la necesidad de adaptarse a un mercado de trabajo con pocas oportunidades profesionales (Santos y Muñoz, 2017). En España, a pesar de que la mayoría de las personas cuentan con estudios superiores, la oferta de cualificaciones no está adaptada a las necesidades del mercado laboral, "de modo que los índices de empleabilidad de los titulados superiores recientes figuran entre los más bajos de Europa, y gran parte de esos titulados desempeñan ocupaciones que no requieren un título universitario" (Comisón Europea, 2016b, p. 6).

La mayoría de los jóvenes universitarios y los adultos resaltaron la oportunidad y la suerte como un requisito necesario para obtener un empleo. En sus representaciones sociales posicionan al empleo como una condición fuera de su dominio, es decir tienen un locus de control externo. El cual se define como una serie de atribuciones sobre sus acciones, de forma tal que cuando algo les sucede, se tienen creencias o suposiciones sobre las causas de lo que está ocurriendo. Dichas causales pueden ser internas (atribuirse a características individuales) o externas (atribuirse al ambiente) (González-Rivera, 2016). Lo que lleva a reconocer otra de las características de las representaciones sociales como guías de actuación en la vida práctica y cotidiana (Jodelet, 2011), en este caso sus prácticas en torno a la búsqueda de empleo podrían reflejar un foco de control externo y limitar con ello la toma de decisiones personales. Al respecto, en un estudio realizado con trabajadores empleados y desempleados en Chile sobre las causas de tener o no tener trabajo, se observó que los empleados tienen un locus de causalidad interno, lo que les permite reforzar la autoestima, autoconcepto, bienestar, valorización de su esfuerzo personal y de sus habilidades. Además, de percepción de estabilidad laboral y control más alto que los par- 
ticipantes desempleados (Moyano et al., 2013). Sin embargo, esta condición no es fija, sino que podría estar influenciada por variables situacionales (Mayora-Pernía y Fernández, 2015).

Los participantes incluyeron en las representaciones sociales del empleo aspectos negativos, tanto individuales como sociales. Por tanto, el trabajo puede contribuir al desarrollo integral de la persona u obstaculizarlo (Serrano-Argüeso, 2015). El empleo puede ser una actividad que no es agradable, ya que se realiza en un lugar específico, con la supervisión de otra persona y también implica cargas físicas y mentales (Da Rosa et al., 2011). El señalamiento de aspectos negativos individuales muestra que el empleo actual también tiene un impacto en la identidad. En una investigación realizada por Sisto y Fardella (2013) con adultos jóvenes chilenos de entre 25 y 34 años de edad, se observó un desplazamiento de los valores laborales hacia el área individual, ya que el valor del trabajo es determinado por la exaltación y realización del individuo. Es decir, hay debilitamiento del sentido colectivo, la experiencia del trabajo como empresa individual y con la pérdida del trabajo como articulador de vínculo social (Sisto y Fardella, 2013). Con esto se logra otra de las funciones de las representaciones sociales ya que "justifican las decisiones y conductas que se manifiestan en las interacciones sociales" (Materán, 2008, p. 245), de tal forma que legitiman el orden social establecido.

En cuanto los aspectos sociales negativos del empleo se pueden encontrar elementos de precariedad en el trabajo. Según la Oficina Internacional del Trabajo (2012) las condiciones de precariedad laboral incluyen un salario bajo, escasa protección frente al despido, falta de acceso a la protección social y a los beneficios que se suelen asociar con un empleo estándar de tiempo completo y posibilidad limitada o nula de los trabajadores de ejercer sus derechos en su trabajo. Además, implica un aumento de desigualdades laborales y sociales que afectan más a poblaciones vulnerables: jóvenes, mujeres, personas con menor nivel de cualificación, inmigrantes y trabajadores mayores de 45 años con bajos niveles de formación (Serrano-Argüeso, 2015). En el caso de los jóvenes se asocia a la mayor frecuencia de la temporalidad y de empleos de tiempo parcial, y al menor poder adquisitivo como producto del menor salario en el trabajo desempeñado. Las mujeres jóvenes presentan mayores porcentajes de contratos a tiempo parcial y suelen recibir menores salarios que los hombres (Echaves y Echaves, 2017). Según la 6 ${ }^{a}$ Encuesta Nacional Española de Condiciones de Trabajo se destaca la elevada precariedad en la inserción laboral de los trabajadores más jóvenes. Ellos acumulan gran parte de la temporalidad del mercado de trabajo, así como de la creciente tasa de contratación a tiempo parcial (Pinilla et al., 2016). A lo que se aúna la división entre los roles de género en el mercado laboral, que fomenta para las mujeres empleos en sectores relacionados con el cuidado, la sanidad, la educación, los servicios sociales, cuidado a dependientes y tareas domésticas (Alcañiz, 2015). Con la precariedad laboral también se dejan de lado los derechos individuales o colectivos, y se promueve la idea de que los individuos deben ser empresarios, lo que acentúa el individualismo ya citado por Sisto y Fardela (2013). Las personas tienen que considerarse a sí mismas como si fueran una empresa, lo cual implica aprender a desarrollar capacidades, competencias y cualidades para mejorar su valor de mercado (Castro, 2019). Es una forma de ser trabajador independiente, con responsabilidad individual, a la vez que se mantienen vinculados al mercado laboral (Sisto y Fardela, 2013).

Las diferencias observadas por los distintos grupos de edad son que los estudiantes de bachillerato tienen una visión idealizada del empleo, del cual esperan obtener ganancias personales, de formación y experiencia. Los jóvenes universitarios destacan la formación académica como principal requisito para 
obtener un empleo, es decir que su condición de estudiantes determina sus prioridades en torno a su representación social del empleo. La población adulta priorizó los beneficios personales y los aspectos negativos del empleo, por tanto cuentan con experiencia en la realización de actividad laboral remunerada, identifican tanto lo positivo como lo negativo de ello. Así, las representaciones sociales del empleo en cada grupo poblacional "propician la comunicación, promueven el pensamiento colectivo y la identidad social" (Materán, 2008, p. 245).

En cuanto a las diferencias culturales observadas por contexto, si bien se obtuvieron las mismas categorías temáticas, muestran variaciones al interior de las mismas, al otorgar pesos diferenciados a cada una de ellas. La población mexicana manifiesta estar relativamente complacida con su realidad laboral, el tener un empleo es satisfactorio, aun cuando podría tener una o más condiciones precarias. La población ecuatoriana muestra una postura más tradicional sobre el empleo al ubicarlo en empresas e instituciones, ello refleja una condición de bienestar dependiente del Estado en el momento de la realización del estudio, dentro de su contexto político y social. Los participantes colombianos cuestionan sus condiciones laborales personales, son críticos ante los tipos de empleos con los que cuentan, sin embargo, no hacen alusiones de tipo social. Así, se observa que la población latinoamericana atribuye una alta valoración a tener un empleo, a pesar de que este puede ser en condiciones de precariedad laboral (Weller y Roethlisberger, 2012).

La población española muestra una perspectiva crítica y social en sus representaciones sociales del empleo, en particular los habitantes del País Vasco, además de señalar más aspectos negativos que los grupos latinoamericanos. Esto podría explicarse con los resultados de la $6^{a}$ Encuesta Nacional Española de Condiciones de Trabajo ya mencionada (Pinilla et al., 2016), donde los trabajadores reportaron que la confianza en encontrar otro empleo de salario similar decrece con la edad, consideran que es más difícil encontrar otro empleo con el mismo salario que recibían en ese momento, perciben un estado de salud más deficiente conforme avanza la edad y reportan que el 27\% tiene jornadas laborales diarias de más de 10 horas (Pinilla et al., 2016). Las diferencias en las representaciones sociales sobre el empleo observadas en estos contextos llevan a establecer maneras de conocimiento distintivas y a la vez compartidas por un grupo cultural determinado (Materán, 2008).

Las representaciones sociales de diferentes tipos de población y grupos de edad sobre el empleo facilitan comprender sus conocimientos sobre el tema y sus experiencias vitales, considerando cómo su contexto cultural condiciona sus percepciones, concepciones y atribuciones. Cabe aclarar que no se pretende hacer generalizaciones a toda la población a partir del estudio de las representaciones sociales de grupos específicos de participantes, ya que los hallazgos aquí mostrados se circunscriben únicamente a estos. Mas sí constituyen una muestra representante más que representativa (en el sentido estadístico) de la población al ser "expertos culturales" de su propio contexto (Weller, 2007, p. 344). Así, identificar la visión de la población facilita el acceso a su mundo de significados, y con ello contar con bases para establecer propuestas de intervención en diferentes niveles.

Este estudio propone el uso de las técnicas metodológicas como los listados de asociación libre como una opción rápida que genera información abundante de la perspectiva de las personas, implican poco tiempo de aplicación y los participantes suelen cooperar de inmediato. Así la transferibilidad metodológica (Flick, 2014) se sugiere para realizar investigaciones en otras áreas del conocimiento. 


\section{Referencias}

Abric, J. C. (2001). Prácticas y representaciones sociales. Ediciones Coyoacán.

Aguilera-Guzmán, R. M., Mondragón, L. y Medina-Mora, M. E. (2008). Consideraciones éticas en intervenciones comunitarias: la pertinencia del consentimiento informado. Salud Mental, 31(2), 129-138.

Alcañiz, M. (2015). Nuevas desigualdades en contextos de crisis: la precariedad laboral femenina en España. En M. M. Serrano (coord.), First International Meeting Of Issow. Work, Social Change and Economic Dynamics: Challenges for Contemporary Societies (pp. 507-526). Escola Superior de Educação - Instituto Politécnico de Lisboa.

Amador, I., Botero, N., Larrahondo, L. y Andrade, V. (2019). Significados del trabajo en mujeres que realizan trabajos productivo y reproductivo. Psicogente, 22(41), 1-36. https://doi.org/10.17081/ psico.22.41.3302

Banch, M. A. (2000). Aproximaciones procesuales y estructurales al estudio de las representaciones sociales. Papers on social representations, (9), 3.1-3.15.

Bernard, H. R. (2006). Research Methods in cultural Anthropology. Altamira Press.

Cerrato, J., Villarreal, M., Ugarteburu, I., Apodaka, E. y Rubio, E. (2003). Nuevas prácticas de trabajo, representaciones sociales del trabajo e identidad social en la sociedad postindustrial. Revista de Psicología Social Aplicada, 13(2),77-119.

Comisión Europea (2016a). Documento de trabajo de los servicios de la Comisión. Informe sobre España 2016, con un examen exhaustivo relativo a la prevención y la corrección de los desequilibrios macroeconómicos, 78. https://ec.europa.eu/info/sites/default/files/cr_spain_2016_es.pdf

Comisión Europea (2016b). Recomendación del Consejo relativa al Programa Nacional de Reformas de 2016 de España y por la que se emite un dictamen del Consejo sobre el Programa de Estabilidad de 2016 de España, 329. https://studylib.es/doc/4626618/recomendaci\%C3\%B3n-de-lacomisi\%C3\%B3n-europea

Da Rosa, S., Chalfin, M., Baasch, D. y Soares-Cugnier, J. (2011). Sentidos y significados del trabajo: un análisis con base en diferentes perspectivas teórico-epistemológicas en Psicología. Universitas Psychologica, 10(1), 175-188.

De Castro, C. (2019). Presentación. La precariedad laboral y más allá. Cuadernos de Relaciones Laborales, 37(1), 11-29. http://dx.doi.org/10.5209/CRLA.63817

Echaves, A. y Echaves, C. (2017). Jóvenes aún más precarios: crisis económica y desigualdad laboral en España. Cuadernos de Investigación en Juventud, (2), 1-19. https://doi.org/10.22400/cij.2.e007

Flick, U. (2014). La gestión de la calidad en Investigación cualitativa. Editorial Morata.

González-Rivera, I. (2016). Creer para poder: la desesperanza aprendida y la autoeficacia en la vida cotidiana. Revista Digital Universitaria, 17(2), 1-8.

Isacovich, P. (2015). Políticas para la inserción laboral de jóvenes: estudios en Latinoamérica y Argentina. Revista Latinoamericana de Ciencias Sociales, Niñez y Juventud, 13(2), 893-905. https://doi.org/10. 11600/1692715x.13224120214

Jodelet, D. (2011). Aportes del enfoque de las representaciones sociales al campo de la educación. Espacios en Blanco, Serie indagaciones, (21), 133-154. 
Kornblit, A. L. (2004). Representaciones sociales y valores de los jóvenes argentinos en relación con el trabajo. Monografías virtuales de la Organización de Estados Iberoamericanos para la Educación, la Ciencia y la Cultura (OEI). https://www.oei.es/historico/valores2/monografias/monografia04/ reflexion04.html

Larrañaga, M., Valencia, J. y Vergès, P. (2007). Representaciones sociales y desempleo: un estudio sobre las contradicciones y especificidades del desempleo femenino. En T. Rodríguez y M. L. García (Eds.), Representaciones sociales: teoría e investigación (pp. 311-328). Universidad de Guadalajara.

Longo, M. E. (2003). Representaciones sociales en torno al trabajo e identidad en varones pobres. [Resumen de presentación en conferencia]. $6^{\circ}$ Congreso Nacional de Estudios del trabajo. Los trabajadores y el trabajo en la crisis. Asociación Argentina de Especialistas en estudios del trabajo (ASET), Buenos Aires, Argentina. https://www.aset.org.ar/congresos/6/archivosPDF/grupoTematico04/023.pdf

Márquez, E., Friemel, E. y Rouquette, M. L. (2005). Valores del trabajo y representaciones sociales. Un estudio exploratorio. Trayectorias, 7(18), 17-32.

Martínez-González, L. M. (2011). Teoría de las Representaciones Sociales: aportes metodológicos a la investigación sobre el homoerotismo. Prospectiva. Revista de trabajo Social e Intervención Social, (16), 1-17. https://doi.org/10.25100/prts.v0i16.1168

Materán, A. (2008). Las representaciones sociales: un referente teórico para la investigación educativa. Geoenseñanza, 13(2), 243-248.

Mayora-Pernía, C. A. y Fernández, N. (2015). Locus de control y rendimiento académico en educación universitaria: Una revisión bibliográfica. Revista Electrónica Educare, 19(3), 1-23. http://dx.doi. org/10.15359/ree.19-3.16

Methivier, J. (2012). État émotionnel négatif et organisation des représentations sociales du travail et du chômage de jeunes adultes en recherche d'emploi. Les Cahiers Internationaux de Psychologie Sociale, 3-4(95-96), 417-437. https://doi.org/10.3917/cips.095.0417

Minayo, M. C. (1995). El desafío del conocimiento. Investigación cualitativa en salud. Editorial Lugar.

Mora, M. (2002). La teoría de las Representaciones Sociales de Serge Moscovici. Athenea Digital, (2), 1-27. https://doi.org/10.5565/rev/athenead/v1n2.55

Moyano, E., Gutiérrez, D., Zúñiga, K. y Cornejo, F. (2013). Empleados y desempleados, atribuciones causales y bienestar subjetivo. Psicologia \& Sociedade, 25(2), 440-450.

Muhr, T. (1997). Scientific software development's atlas-ti. Visual qualitative data. Versión 7. Scientific Software Development. https://atlasti.com/

Navarro, J. (2007). Experiencias y representaciones sociales del trabajo en jóvenes. En T. Rodríguez y M. L. García (Eds.), Representaciones sociales: teoría e investigación (pp. 283-309). Universidad de Guadalajara.

Organización Internacional del Trabajo. (2012). Del trabajo precario al trabajo decente. Documento final del simposio de los trabajadores sobre políticas y reglamentación para luchar contra el empleo precario. Oficina de Actividades para los Trabajadores (ACTRAV). http://www.ilo.org/wcmsp5/ groups/public/---ed_dialogue/---actrav/documents/meetingdocument/wcms_179789.pdf

Organización Internacional del Trabajo (OITa). (2017). Informe Mundial sobre Salarios 2016/2017: La desigualdad salarial en el lugar de trabajo. https:/www.ilo.org/global/publications/books/ WCMS_541632/lang--es/index.htm 
Organización Internacional del Trabajo (OITb). (2017) Panorama Laboral 2017. https://www.ilo.org/ americas/publicaciones/WCMS_613957/lang--es/index.htm

Oficina Internacional del Trabajo. (2018). Tesauro de la Oficina Internacional del Trabajo. Biblioteca de la Oficina Internacional del Trabajo. https:/www.ilo.org/inform/online-information-resources/ terminology/thesaurus/lang--es/index.htm

Oficina Internacional del Trabajo. (2019). Perspectivas Sociales y del Empleo en el Mundo: Tendencias 2019. https://www.ilo.org/global/research/global-reports/weso/2019/lang--es/index.htm3

Oficina Internacional del Trabajo. (2020). World Employment and Social Outlook: Trends 2020. https:// www.ilo.org/wcmsp5/groups/public/---dgreports/---dcomm/---publ/documents/publication/ wcms_734455.pdf

Pereira, L. (2013). La representación social del trabajo en jóvenes universitarios. Revista Latinoamericana de derecho Social, (17), 145-177.

Pérez-Rubio, A. M. (2004). Los jóvenes y el trabajo. Un estudio sobre representaciones sociales. Monografías virtuales de la Organización de Estados Iberoamericanos para la Educación, la Ciencia y la Cultura (OEI). https://www.oei.es/historico/valores2/monografias/monografia04/reflexion03.htm

Pinilla, J., Almodóvar, A., Hervás, P., Blanco, L. y Zimmermann, M. (2016). La edad de las condiciones de trabajo. Resultados de la $6^{a}$ EWCS-España. Seguridad y Salud en el Trabajo. Seguridad y Salud en el Trabajo, 88, 15-28. https:/www.insst.es/documents/94886/175912/ N\%C3\%BAmero+88+(versi\%C3\%B3n+pdf

Ruiz, J. I., Ponce De León, E., Herrera, A. N., Jiménez, H. y Medellín, E. (2001). Avances en Medición Evaluación en Psicología y Educación. Universidad del Bosque.

Santos, A. y Muñoz, D. (2017). Más allá de la precariedad laboral. Los nuevos rasgos de la precariedad juvenil. Gaceta Sindical. Reflexión y debate, (29), 235-252.

Scheffer-Garay, A. B. (2011). As representações sociais de jovens participantes de projeto social de inserção no mercado de trabalho. REGE, 18(1), 93-109.

Sibaja, I. (2013). Consideraciones teóricas: la salud sexual y la prevención de infecciones de transmisión sexual en mujeres. Rev. Reflexiones, 92(2), 79-90.

Serrano-Argüeso, M. (2015). Medidas de reparto de empleo en España en un contexto de crisis económica: ¿solución contra el desempleo o vía de incremento de la precariedad laboral?. Revista Internacional y Comparada de Relaciones Laborales y Derecho del Empleo, 3(3), 120-146.

Simón, J. (2015). Las expectativas de búsqueda del primer empleo de universitarios con formación híbrida. El caso de una universidad pública mexicana. Cuadernos de Investigación Educativa, 6(2), 33-49. http://dx.doi.org/10.18861/cied.2015.6.2.35

Sisto, V. y Fardella, C. (2013). Rearmando el Trabajo. Significados del trabajo en tiempos de flexibilización laboral. En P. Vidal y M.A. Rodríguez (Eds.), Transformaciones sociales: la precariedad laboral puesta en cuestión (pp. 127-145). Editorial Espacio.

Suri, H. (2011). Purposeful Sampling in Qualitative Research Synthesis. Qualitative Research Journal, 11(2), 63-75. https://doi.org/10.3316/QRJ1102063

Torres-López, T. M. y Díaz-Villanueva, A. I. (2016). Oportunidad, precariedad y estrés: Representaciones sociales de empleo y desempleo de jóvenes universitarios de una universidad pública de Guadalajara, México. En N. González y L.E. Benhumea (Eds.), Salud, trabajo y género (pp. 83-106). Editorial Porrúa. 
Torres-López, T. M., Acosta-Fernández, M. y Aguilera-Velasco, M. A. (2017). Preparación para el trabajo: Representaciones sociales del empleo y desempleo de jóvenes mexicanos sin trayectoria laboral. Revista Educación y Desarrollo Social, 11(1), 85-101. https://dx.doi.org/10.18359/reds.1867

Torres-López, T. M., Munguía, J. A. y Torres-Valdovinos, M. M. (2018a). Representaciones Sociales de empleo y desempleo en estudiantes universitarios de Quito, Ecuador. Revista Reflexiones, 97(2), 7-22.

Torres-López, T. M., Munguía-Cortés, J. A. y Soltero-Avelar, R. (2018b). Representaciones sociales de los conceptos de empleo y desempleo de habitantes de Santa Cruz, Tenerife. Quaderns de Psicologia, 20(2), 1-14. http://dx.doi.org/10.5565/rev/qpsicologia.1438

Torres-López, T. M., Quezada-Valadez, M. G. y Herrera-Pérez, J. I. (2018c). Representaciones sociales de empleo y desempleo de trabajadores de la salud de Bogotá, Colombia y de Morelia, México. Rev. Fac. Cienc. Salud UDES, 5(2), 25-33. http://dx.doi.org/10.20320/rfcsudes.v5i2.104

Torres-López, T. M., Lozano-González. A. F. y Acosta-Fernández, M. (2018d). Representaciones sociales del empleo y desempleo de población del País Vasco. Apuntes de Psicología, 36(3), 155-165.

Torres-López, T. M., Acosta-Fernández, M. y Parra-Osorio, L. (2019). Dimensiones culturales del empleo y desempleo de jóvenes universitarios de Cali, Colombia. Psicogente, 22(41), 1-23. https://doi. org/10.17081/psico.22.41.3306

Vesga, J. J. (2017). Conceptualización en la psicología organizacional y del trabajo: necesidad de congruencia con fenómenos y hechos. Quaderns de Psicologia, 19(1), 89-100. http:/doi.org/10.5565/rev/ qpsicologia.1385

Weller, J. y Roethlisberger, C. (2012). La calidad del empleo en América Latina: un análisis de conjunto. En S. Farné (Ed.), La calidad del empleo en el siglo XXI (pp. 33-118). Universidad Externado de Colombia.

Weller, S. (2007). Questions Cultural Consensus Theory: Applications and Frequently Asked. Field Methods, 19(4), 339-368. https://doi.org/10.1177/1525822X07303502 